\title{
Fast variance image predictions for quadratically regularized statistical image reconstruction in fan-beam tomography
}

\author{
Yingying Zhang ${ }^{1}$, Student member, IEEE, Jeffrey A. Fessler ${ }^{1}$, Senior member, IEEE, Jiang Hsieh ${ }^{2}$, Member, IEEE \\ yzz,fessler@eecs.umich.edu, Jiang.Hsieh@med.ge.com
}

\begin{abstract}
Accurate predictions of variance can be useful for algorithm analysis and for the design of regularization methods. Computing predicted variances at every pixel using matrix-based approximations [1] is impractical. Even the recently adopted methods that are based on local discrete Fourier approximations are impractical since they would require two 2D FFT calculations for every pixel, particularly for shift-variant systems like fan-beam tomography. This paper describes a new analytical approach to predict the approximate variance maps of images reconstructed by penalized likelihood estimation with quadratic regularization in a fan-beam geometry. This analytical approach requires computation equivalent to one backprojection and some simple summations, so it is computationally practical even for the data sizes in X-ray CT. Simulation results show that it gives accurate predictions of the variance maps. The parallel-beam geometry is a simple special case of the fan-beam analysis.
\end{abstract}

Index Terms - variance approximation, local discrete Fourier analysis, fan-beam tomography.

\section{INTRODUCTION}

$\mathbf{S}$ TATISTICAL methods have obtained increasing attention in tomography image reconstruction for the improved noise and resolution properties. These methods are usually nonlinear and shift-variant. To analyze the statistical characteristics of the reconstructed images, one would often like to predict the variance of pixel values. The variance information provides an uncertainty measure of the reconstructed image and may be useful in regularization parameter selection.

We focus here on penalized likelihood estimators obtained by minimizing a cost function

$$
\hat{\mu}=\underset{\mu}{\arg \min } \Phi(\mu, \boldsymbol{Y}) .
$$

The cost function includes a log-likelihood term and a regularization term:

$$
\Phi(\mu, \boldsymbol{Y})=-L(\mu, \boldsymbol{Y})+\beta R(\mu) .
$$

For monoenergetic transmission tomography under the Poisson noise model, the log-likelihood is

$$
L(\mu, \boldsymbol{Y})=\sum_{i} Y_{i} \log \left(\bar{Y}_{i}\right)(\mu)-\bar{Y}_{i}(\mu)
$$

This work is supported in part by NIH/NCI grant P01 CA87634 and GE Medical Systems.

${ }^{1}$ EECS Dept., The University of Michigan; ${ }^{2}$ GE Healthcare Technologies where

$$
\bar{Y}_{i}=b_{i} \mathrm{e}^{-[\boldsymbol{A} \mu]_{i}}+r_{i},
$$

where $\boldsymbol{A}$ is the system matrix, $b_{i}$ denotes the blank scan, and $r_{i}$ denotes the additive contribution of scatter. The goal is to estimate the attenuation image $\mu$ from the data $\boldsymbol{Y}$.

We focus here on quadratic regularization for which

$$
R(\mu)=\frac{\beta}{2} \mu^{\prime} \boldsymbol{R} \mu,
$$

where $\boldsymbol{R}$ denotes the Hessian of the penalty function.

In [1], the following approximation to the covariance of $\hat{\mu}$ was derived:

$$
\operatorname{Cov}\{\hat{\mu}\} \approx\left(\boldsymbol{A}^{\prime} \boldsymbol{W} \boldsymbol{A}+\beta \boldsymbol{R}\right)^{-1} \boldsymbol{A}^{\prime} \boldsymbol{W} \boldsymbol{A}\left(\boldsymbol{A}^{\prime} \boldsymbol{W} \boldsymbol{A}+\beta \boldsymbol{R}\right)^{-1},
$$

using the implicit function theorem, the Taylor expansion and the chain rule. The matrix method described in (1) has been used in various applications, [2], [3]. Simulation and experimental results show the accuracy of this covariance approximation in image regions where the nonnegativity constraint is usually inactive. To accelerate variance computation, circulant approximations are usually used in practice, as follows:

$$
\operatorname{Var}\left\{\hat{\mu}_{j}\right\} \approx \sum_{k} \frac{\mathcal{F}\left(\boldsymbol{A}^{\prime} \boldsymbol{W} \boldsymbol{A} e_{j}\right)_{k}}{\left[\mathcal{F}\left(\boldsymbol{A}^{\prime} \boldsymbol{W} \boldsymbol{A} e_{j}\right)_{k}+\mathcal{F}\left(\beta \boldsymbol{R} e_{j}\right)_{k}\right]^{2}},
$$

where $\mathcal{F}$ is a discrete Fourier Transform and $e_{j}$ is the $j$ th unit vector of length $p$. Evaluating this expression requires two FFTs per pixel.

The computation of this DFT approximation are still expensive for realistic image size when the variance must be computed for all pixels, particularly for shift-variant systems like fan-beam tomography.

In this paper, we propose to return to the continuous space and use "local Fourier analysis" to approximate the variance of the fan-beam reconstructed tomographic images, based on a key concept from previous work, local shift invariance [4]-[6] and the relationship between the fan-beam and parallel-beam coordinates.

The paper is organized as follows. Section II briefly describes the general analytical approach for the variance approximation for fan-beam tomography. Section III applies this method to the quadratic regularization case, particularly a standard quadratic 
penalty as an example. Section IV gives simulation results, including an comparison of the analytical, FFT-based and empirical standard deviation images. Finally, discussion and conclusions are in Section V.

\section{The Analytical Variance Approximation}

With the same philosophy in [1], one can derive the continuous-space counterpart of equation (1),

$$
\operatorname{Cov}\{\hat{\mu}\} \approx\left(\mathcal{A}^{*} \mathcal{W} \mathcal{A}+\beta \mathcal{R}\right)^{-1} \mathcal{A}^{*} \mathcal{W} \mathcal{A}\left(\mathcal{A}^{*} \mathcal{W} \mathcal{A}+\beta \mathcal{R}\right)^{-1},
$$

where $\mathcal{A}$ is the projection operator, $\mathcal{W}$ is the fan-beam weighting operator $(\mathcal{W} p)(\sigma, \beta)=w(\sigma, \beta) p(\sigma, \beta)$ and $\mathcal{R}$ is the regularization operator.

Let $K\left(x, y ; x^{\prime}, y^{\prime}\right)=\operatorname{Cov}\left\{\hat{\mu}(x, y), \hat{\mu}\left(x^{\prime}, y^{\prime}\right)\right\}$ denote the spatial autocovariance function of $\hat{\mu}(x, y)$. Based on the fact that the statistics in $\mathcal{W}$ vary smoothly spatially, we make the following "local stationarity" approximation of $K$ around a point $\left(x_{j}, y_{j}\right)$ :

$$
\begin{aligned}
K\left(x, y ; x^{\prime}, y^{\prime}\right) & \approx K^{j}\left(x-x^{\prime}, y-y^{\prime}\right) \\
K^{j}\left(\triangle_{x}, \triangle_{y}\right) & \triangleq K\left(x_{j}+\triangle_{x}, y_{j}+\triangle_{y} ; x_{j}, y_{j}\right) .
\end{aligned}
$$

Using this approximation along with local Fourier analysis, the local covariance operator near $\left(x_{j}, y_{j}\right)$ can be expressed as

$$
\begin{aligned}
\mathcal{K}_{\hat{\mu}}^{j} & \triangleq \operatorname{Cov}\{\hat{\mu}\} \\
& =\mathcal{F}_{2}^{-1} \mathcal{D}\left(\frac{H_{j}(\rho, \Phi)}{\left(H_{j}(\rho, \Phi)+\beta R_{j}(\rho, \Phi)\right)^{2}}\right) \mathcal{F}_{2},
\end{aligned}
$$

where $H_{j}(\rho, \Phi)$ is the local frequency response of the Gram operator $\mathcal{A}^{*} \mathcal{W} \mathcal{A} \delta_{j}$ and $R_{j}(\rho, \Phi)$ is the local frequency response of $\mathcal{R} \delta_{j}$, where $\delta_{j}(x, y) \triangleq \delta\left(x-x_{j}, y-y_{j}\right)$. The variance at the location of interest $\left(x_{j}, y_{j}\right)$ is given by

$$
\begin{aligned}
\operatorname{Var}\left\{\hat{\mu}_{j}\right\} & =\left\langle\delta_{j}, \mathcal{K}_{\hat{\mu}}^{j} \delta_{j}\right\rangle \\
& =\left\langle\mathcal{F} \delta_{j},\left(\frac{H_{j}(\rho, \Phi)}{\left(H_{j}(\rho, \Phi)+\beta R_{j}(\rho, \Phi)\right)^{2}}\right)\right\rangle \\
& =\int_{0}^{2 \pi} \int_{0}^{\infty} \frac{H_{j}(\rho, \Phi) \rho \mathrm{d} \rho \mathrm{d} \Phi}{\left[H_{j}(\rho, \Phi)+\beta R_{j}(\rho, \Phi)\right]^{2}} .
\end{aligned}
$$

This is a general expression for predicting the variance of a penalized-likelihood estimator for any geometry.

We focus on fan-beam CT here. Let $h_{j}(x, y)$ be the impulse response of the fan-beam Gram operator $\mathcal{A}^{*} \mathcal{W} \mathcal{A}$, defined as follows:

$$
h_{j}(x, y) \triangleq\left(\mathcal{A}^{*} \mathcal{W} \mathcal{A} \delta_{j}\right)(x, y) .
$$

Ignoring detector response, one can show that (4) can be expressed in polar coordinates $(r, \varphi)$ as

$$
h_{j}(r, \varphi)=\frac{1}{|r|} w_{j}(\varphi+\pi / 2),
$$

where the angular dependent weighting $w_{j}(\varphi)$ is given by

$$
w_{j}(\varphi)=\left.w\left(\sigma^{\prime}, \beta^{\prime}\right) J\left(\sigma^{\prime}\right)\right|_{\varphi^{\prime}=\varphi}+\left.w\left(\sigma^{\prime}, \beta^{\prime}\right) J\left(\sigma^{\prime}\right)\right|_{\varphi^{\prime}=\varphi-\pi} .
$$

$J(\sigma)$ is the determinant of the Jacobian matrix of transforming from the fan-beam coordinates to parallel-beam coordinates:

$$
J\left(\sigma^{\prime}\right)=1 /\left|D_{s o} \cos \gamma\left(\sigma^{\prime}\right) \dot{\gamma}\left(\sigma^{\prime}\right)\right|,
$$

where $D_{s o}$ is the distance from source to rotation center and $\gamma\left(\sigma^{\prime}\right)=\sigma^{\prime} / D_{s d}$ for the equiangular case. $D_{s d}$ is the distance from source to detector.

Thus, we can obtain the local frequency response of the Gram operator by taking local Fourier transform of $h_{j}(r, \varphi)$ in equation (5):

$$
\begin{aligned}
H_{j}(\rho, \Phi) & =\int_{0}^{\pi} \int_{-\infty}^{\infty} h_{j}(r, \varphi) \mathrm{e}^{-\imath 2 \pi r \rho \cos (\varphi-\Phi)}|r| \mathrm{d} r \mathrm{~d} \varphi \\
& =\int_{0}^{\pi} w_{j}(\varphi+\pi / 2) \int_{-\infty}^{\infty} \delta(\rho \cos (\varphi-\Phi)) \mathrm{d} \varphi \\
& =\frac{1}{|\rho|} \int_{0}^{\pi} w_{j}\left(\varphi^{\prime}\right) \delta\left(\sin \left(\varphi^{\prime}-\Phi\right)\right) \mathrm{d} \varphi^{\prime} \\
& =\frac{1}{|\rho|} w_{j}(\Phi) .
\end{aligned}
$$

The local Fourier transform is used as an analysis tool in our derivation of $H_{j}(\rho, \Phi)$. However, there is no need to take any Fourier transform in our computation!

In fan-beam tomography, detector blur effects depend on the distances between each image pixel and the detector elements, and hence cannot be modeled exactly in the frequency domain. We approximate the depth-dependent detector response by an effective beam width $\left(D_{s o} / D_{s d}\right) \triangle_{s}$ at the rotation center, where $\triangle_{s}$ is the detector spacing. For simplicity, we model the frequency response of this effective detector blur as $C \operatorname{rect}\left(\rho / 2 \rho_{\max }\right)$, where $\rho_{\max }=\frac{D_{s d}}{2 D_{s o} \triangle_{s}}$. The local frequency response of the Gram operator becomes

$$
H_{j}(\rho, \Phi)=\frac{C \operatorname{rect}\left(\rho / 2 \rho_{\max }\right)}{|\rho|} w_{j}(\Phi) .
$$

Substituting $H_{j}(\rho, \Phi)$ into (3), the variance of $\hat{\mu}_{j}$ can be approximated analytically as

$$
\operatorname{Var}\left\{\hat{\mu}_{j}\right\} \approx \int_{0}^{2 \pi} \int_{0}^{\rho_{\max }} \frac{\frac{C w_{j}(\Phi)}{|\rho|}}{\left(\frac{C w_{j}(\Phi)}{|\rho|}+\beta R_{j}(\rho, \Phi)\right)^{2}} \rho \mathrm{d} \rho \mathrm{d} \Phi .
$$

The parallel-beam geometry is just a special case with the angular weighting function consisting only of the data statistics $w\left(\sigma^{\prime}, \beta^{\prime}\right)$.

\section{Quadratically PENAlized-LiKelihood ESTIMATOR}

Consider the quadratic regularization design for fan-beam tomography whose $R(\rho, \Phi)$ is approximately separable in $\rho$ and $\Phi$,

$$
R_{j}(\rho, \Phi) \approx(2 \pi \rho)^{2} \tilde{R}_{j}(\Phi)
$$


The variance approximation in (6) becomes

$$
\begin{aligned}
\operatorname{Var}\left\{\hat{\mu}_{j}\right\} & \approx \int_{0}^{2 \pi} \int_{0}^{\rho_{\max }} \frac{\frac{C w_{j}(\Phi)}{|\rho|}}{\left(\frac{C w_{j}(\Phi)}{|\rho|}+\beta(2 \pi \rho)^{2} \tilde{R}_{j}(\Phi)\right)^{2}} \rho \mathrm{d} \rho \\
& =\int_{0}^{2 \pi} \frac{\rho_{\max }^{3} / 3}{\left[C w_{j}(\Phi)+\beta 4 \pi^{2} \rho_{\max }^{3} \tilde{R}_{j}(\Phi)\right]^{2}} \mathrm{~d} \Phi
\end{aligned}
$$

for a quadratic penalty function. The computation of $w_{j}(\Phi)$ for all pixels only requires the same computation time as one backprojection. The integral can be evaluated by a finite summation with correctly chosen $\rho_{\max }$. The variance image prediction in (7) requires much less computation than (1) and thus is practical even for realistic tomographic image sizes.

\section{A. Standard Quadratic Penalty Function}

Consider a standard quadratic penalty where $\tilde{R}_{j}(\Phi)=\tilde{R}_{j}=$ $\kappa_{j}^{2}$ is independent of $\Phi . \tilde{R}_{j}$ is the design parameter that allows different degree of regularization and hence controls the amplitude of PSF.

The variance approximation in this case has a very simple form:

$$
\operatorname{Var}\left\{\hat{\mu}_{j}\right\} \approx \frac{\rho_{\max }^{3}}{3} \int_{0}^{2 \pi} \frac{1}{\left[C w_{j}(\Phi)+\beta 4 \pi^{2} \rho_{\max }^{3} \tilde{R}_{j}\right]^{2}} \mathrm{~d} \Phi
$$

\section{EXAMPLE RESUlTS}

To evaluate the performance of the proposed method, we implemented the above prediction for the fan-beam tomographic images reconstructed by quadratically penalized likelihood algorithm. We simulated 450 realizations of fan-beam transmission scans using $128 \times 128$ Zubal phantom with the corresponding sinogram size is 222 samples in $\sigma$, spaced by $\triangle_{\sigma} \approx 0.248^{\circ}$ and 246 source positions over $360^{\circ} \cdot \rho_{\max }=$ $0.224 \mathrm{~mm}^{-1}$. The regularization parameter $\beta=2^{12}$ is chosen to give $\mathrm{FWHM}=1.76$ pixels, i.e., $6.0 \mathrm{~mm}$ at the center of the image. For each realization, $\hat{\mu}$ is reconstructed using 400 iterations of the incremental optimization transfer algorithm (PL-IOT) of [7] to ensure the convergence. The initial images are the FBP images with equivalent spatial resolutions. The sample standard deviation is then calculated pixel by pixel within the finite support used in reconstruction.

The reconstructed images and standard deviation images are displayed in Fig. 1. For a complete comparison, we also computed the predicted standard deviation using the FFT method along two profiles. The standard deviation image for 450 realizations of FBP image is also computed and displayed in Fig. 1. Note that the values of the FBP standard deviation are approximately 4 times higher than that of the PL-IOT reconstruction. The advantage of the statistical reconstruction methods in the noise and resolution tradeoff has been further confirmed.

The central horizontal and vertical profiles are shown in Fig. 2 and Fig. 3. The analytical, the FFT-based and empirical standard deviations agree with one another very closely within the finite support used in image reconstruction.

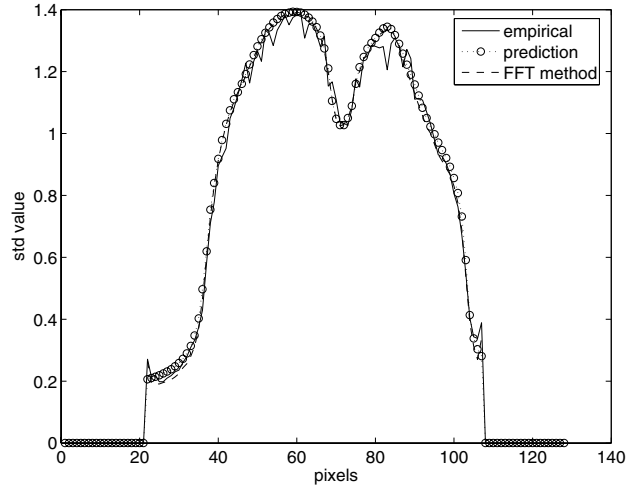

Fig. 2. Central vertical profiles for predicted and empirical standard deviation images shown in Fig. 1.

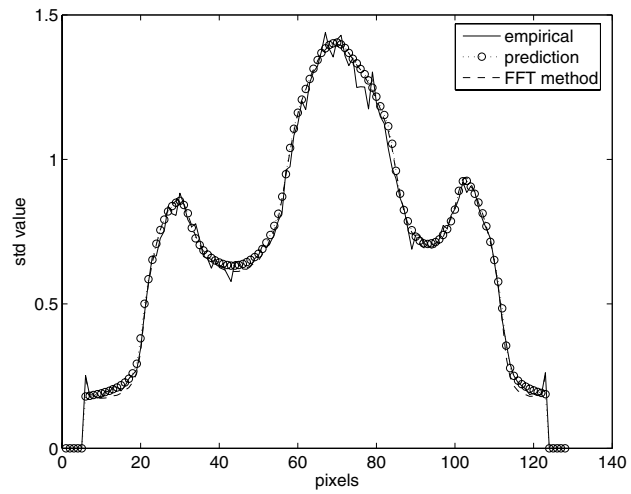

Fig. 3. Central horizontal profiles for predicted and empirical standard deviation images shown in Fig. 1.

\section{Discussion}

In this paper, an analytical variance approximation is given by (3), more particularly by (7) for a quadratically penalized likelihood estimator in fan-beam tomography. The computation of this proposed method is equivalent to one backprojection with an additional summation, and is much less than the previous methods. The empirical result from the simulated fan-beam CT transmission scans demonstrates the proposed variance approximation is very accurate and thus provides a useful means to estimate the variance image for realistic tomographic image size.

Further work is needed to evaluate the proposed variance prediction in a modified quadratic penalty design for fan-beam tomography in [8] that leads to nearly uniform and isotropic spatial resolution. We also need to evaluate the application of the proposed "continuous space" philosophy in covariance matrix approximation which is very useful in detection tasks. Furthermore, we will also investigate how to apply this variance approximation in choosing the regularization parameter for the edge-preserving regularization, possibly a locally-varied $\delta$. Finally, we want to generalize this work to $3 \mathrm{D}$ cone beam tomography. 


\section{true phantom $\quad \mathrm{PL}-\mathrm{IOT}$ reconstruction $\mathrm{FBP}$ with matched resolution}
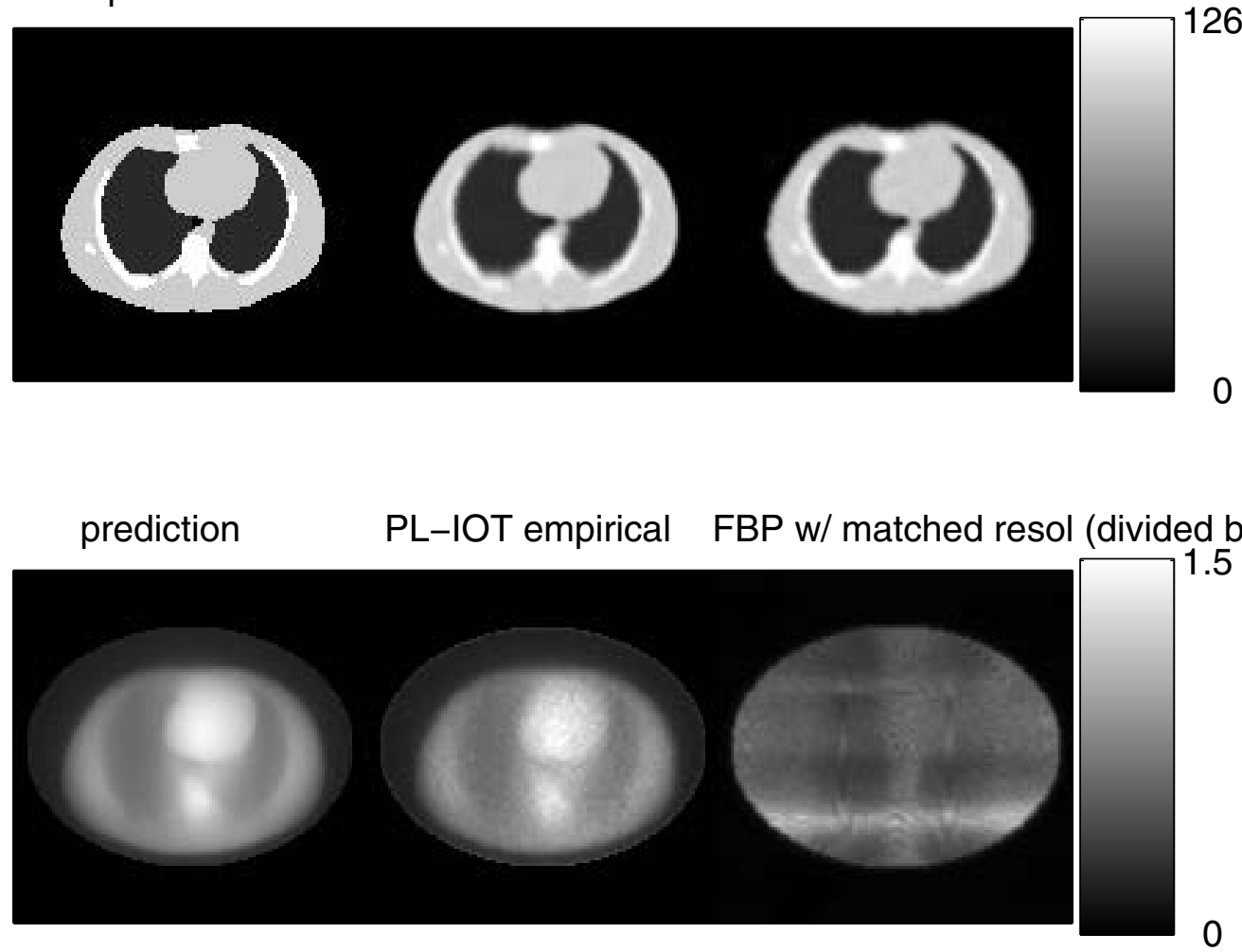

Fig. 1. Predicted and empirical standard deviation images (in HU) for Zabul phantom for PL fan-beam transmission image reconstruction using the standard quadratic penalty.

\section{REFERENCES}

[1] J. A. Fessler, "Mean and variance of implicitly defined biased estimators (such as penalized maximum likelihood): Applications to tomography," IEEE Trans. Im. Proc., vol. 5, no. 3, pp. 493-506, Mar. 1996.

[2] J. Qi and R. H. Huesman, "Theoretical study of lesion detectability of MAP reconstruction using computer observers," IEEE Trans. Med. Imag., vol. 20, no. 8, pp. 815-22, Aug. 2001.

[3] J. W. Stayman and J. A. Fessler, "Efficient calculation of resolution and covariance for fully-3D SPECT," IEEE Trans. Med. Imag., vol. 23, no. 12, pp. 1543-56, Dec. 2004.

[4] J. A. Fessler and S. D. Booth, "Conjugate-gradient preconditioning methods for shift-variant PET image reconstruction," IEEE Trans. Im. Proc., vol. 8, no. 5, pp. 688-99, May 1999.

[5] J. Qi and R. M. Leahy, "A theoretical study of the contrast recovery and variance of MAP reconstructions with applications to the selection of smoothing parameters," IEEE Trans. Med. Imag., vol. 18, no. 4, pp. 293-305, Apr. 1999.

[6] J. W. Stayman and J. A. Fessler, "Regularization for uniform spatial resolution properties in penalized-likelihood image reconstruction," IEEE Trans. Med. Imag., vol. 19, no. 6, pp. 601-15, June 2000.

[7] S. Ahn, J. A. Fessler, D. Blatt, and A. O. Hero, "Convergent incremental optimization transfer algorithms: Application to tomography," IEEE Trans. Med. Imag., 2004, to appear.

[8] H. Shi and J. A. Fessler, "Quadratic regularization design for fan beam transmission tomography," in Proc. SPIE 5747, Medical Imaging 2005: Image Proc., 2005, pp. 2023-33. 